\title{
NT-ProBNP and Cardiac Troponin I In Virulent Canine Babesiosis
}

Remo Lobetti ${ }^{a}{ }^{*}$, Robert Kirberger ${ }^{b}$, Ninette Keller ${ }^{b}$, Frank Kettner $^{b}$, Eran Dvir ${ }^{b}$

${ }^{a}$ Bryanston Veterinary Hospital, Box 67092, Bryanston, South Africa.

${ }^{\mathrm{b}}$ Department of Companion Animal Clinical Studies, Faculty of Veterinary Science, University of Pretoria, Onderstepoort, South Africa.

*Corresponding author. Tel.: +27117066023; fax: +27117065801. E-mail address: rlobetti@mweb.co.za (R. Lobetti).

Current address of Ninette Keller: School of Veterinary and Biomedical Science, James Cook University, Townsville, Australia.

Current address of Frank Kettner: Tygerberg Animal Hospital, Box 4011, Old Oak, Cape Town, South Africa. 


\begin{abstract}
Although cardiac pathology and consequently elevated serum cardiac troponin I (cTnl) have been reported, clinically it remains difficult to diagnose cardiac involvement in canine babesiosis. Thus the use of cardiac biomarkers would be useful in determining if a dog with babesiosis also has concurrent cardiac dysfunction. The objectives of this study were to determine plasma $\mathrm{N}$ terminal brain natriuretic peptide (NT-proBNP) in canine babesiosis and if it is correlated with cTnl. Three groups of dogs with babesiosis were used: mild uncomplicated (Group 1), severe uncomplicated (Group 2), and complicated (Group 3), and a control group (Group 4) with 15 dogs per group. Each animal had the following determined: serum urea and creatinine, urea: creatinine ratio, cystatin-C, cTnl, blood lactate, plasma NT-proBNP, fractional shortening (FS), and blood pressure. The median NT-proBNP value in Groups 1 to 4 was 246, 650,638, and 106 pmol/l. All 3 babesiosis groups had a statistically elevated NT-proBNP level compared to the control group and Groups 2 and 3 showed significantly higher values compared to Group 1. Median cTnl in Group 1 to 3 was $0.39,0.4$, and $1.45 \mathrm{ng} / \mathrm{ml}$, respectively with the control group having concentrations below the detection limit $(0.2 \mathrm{ng} / \mathrm{ml})$. There was a significant difference in cTnl concentration between the control group and group 3 but no statistical difference between the other babesiosis groups.
\end{abstract}

The study concluded that dogs with babesiosis showed elevated levels NT-proBNP and the more severe the disease process the greater the elevation. This elevation is earlier or independent of the increased cTnl.

Keywords: - Cardiac biomarkers; BNP; Troponin; Dog 


\section{Introduction}

Cardiac pathology has been reported in canine babesiosis (Dvir et al., 2004; Lobetti et al., 2002), but remains clinically difficult to diagnose as the signs of acute cardiac dysfunction such as dyspnea and weakness overlap those occurring in canine babesiosis namely, severe anemia and acute respiratory distress syndrome (ARDS). In a dog with babesiosis showing dyspnea and weakness, routine laboratory testing, ECG, and thoracic radiographs have limited sensitivity and specificity in pinpointing cardiac pathology. Thus, the availability of accurate, sensitive, and specific blood tests for heart failure would be useful in determining if a dog that has babesiosis also has concurrent cardiac pathology as a complication. Echocardiography is a potentially more sensitivity test for cardiac pathology, but its use in canine babesiosis has not been reported.

The natriuretic peptides and troponin represent blood-based substances that are associated with cardiac structure, function, and injury. The natriuretic peptides are released by myocardial tissue primarily in response to increased myocardial wall stress whereas troponins are released as a result of myocyte injury or necrosis (Oyama et al., 2007). During development of cardiac disease, the circulatory natriuretic peptides and troponins are all elevated, presumably along a continuum concurrent with disease severity. Therefore, elevation of blood levels of these markers may be found in patients with early stages of nonclinical disease (Oyama et al., 2007).

Natriuretic peptides have been widely demonstrated to be useful as markers of the presence and severity of cardiac disease (Boswood et al., 2003; Boswood et al., 2008; Fine et al., 2008; Haggstrom et al., 2000; Oyama et al., 2008; Prosek et al., 2007). Currently, measurement of NT-proBNP is recommended as one of the first steps in the evaluation of humans suspected to have heart failure, with veterinary clinical studies indicating that NTproBNP can likely be used in a similar manner in dogs (Boswood et al., 2008; Fine et al., 
2008; Oyama et al., 2008; Prosek et al., 2007).

Brain natriuretic peptide (BNP) is secreted by the ventricles in response to an increase in intra-cardiac hydrostatic pressure, increased cardiac wall stress, angiotensin II, myocardial hypoxia, and heightened sympathetic tone (Chetboul et al., 2004; Oyama et al., 2009). In humans plasma BNP concentrations are good indicators for the severity of left ventricular dysfunction that occurs with acute myocardial infarction and congestive heart failure (Asano et al., 1999; De Lemos et al., 2001; Omland et al., 1996).

Brain natriuretic peptide is synthesized as a pre-prohormone and processed to the prohormone form in ventricular myocytes. The NT-proBNP is formed when its parent prohormone, pro-BNP, is cleaved into 2 molecules, namely NT-proBNP and C-BNP. As such, both NT-proBNP and C-BNP concentrations represent potential diagnostic tools in the diagnosis of congestive heart failure (Boswood et al., 2008; Oyama et al., 2008; Oyama et al., 2009). In circulation, C-BNP is rapidly degraded, making laboratory testing difficult, however, NT-proBNP possesses a much longer half-life, making it easier to detect (Oyama et al., 2008; Oyama et al., 2009).

In one study NT-proBNP concentrations were significantly different between healthy control dogs and dogs with cardiac disease, between dogs with cardiac disease with congestive heart failure and dogs with cardiac disease without congestive heart failure, and between dogs with cardiac disease and cardiomegaly and dogs with cardiac disease without cardiomegaly (Oyama et al., 2008). NT-proBNP concentrations have also been shown to discriminate between dogs diagnosed with cardiac disease and those diagnosed with respiratory disease with no evidence of cardiac disease (Boswood et al., 2008), however, dogs with respiratory disease tend to have higher NT-proBNP values and therefore the cut off level should be adjusted. In dogs with cardiomyopathy, BNP, and cardiac troponin I (cTnl) concentrations were significantly increased in dogs with ECG and echocardiographic 
evidence of occult cardiomyopathy, however, only the BNP assay had adequate sensitivity and specificity to be of diagnostic use (Oyama et al., 2007).

Troponins are a sensitive and persistent indicator of cardiac injury (Missov et al., 1997; O'Brein et al., 1997; Rossi, 1999). Various studies have shown that increased cTnl blood concentrations in dogs were consistent with increased severity of cardiac disease and short survival times (Fonfara et al., 2010). Previous studies have showed myocardial damage, ECG changes and elevated CTnl in canine babesiosis (Dvir et al., 2004; Lobetti et al., 2002). Dogs with babesiosis that showed histological myocardial lesions in one study (Dvir et al., 2004) had elevated cardiac troponin concentrations (Lobetti et al., 2002). Although the numbers were small, the results of that study suggested that an increased cTnl concentration was associated with a poor outcome.

The most common cardiac pathology in canine babesiosis is micro-infarction and inflammation (Dvir et al., 2004). Myocardial infarction can result in vascular pooling and myocardial depression (Collinson et al., 2000), which may lead to hypotension. Hypotension has been reported in canine babesiosis (Jacobson et al., 2000), and the presence and severity of hypotension increases with increased disease severity and a feasible explanation for the hypotension in canine babesiosis would be myocardial pathology.

Myocardial injury is common in complicated but rare in uncomplicated malaria (Ehrhardt et al., 2005). In humans with severe falciparum malaria, it has been shown that there is myocardial impairment as defined by elevated levels of circulation cardiac markers (Ehrhardt et al., 2004; Ehrhardt et al., 2005). An added benefit of these markers is that the assay is not affected by bilirubinemia, hemoglobinemia, and hyperlipemia (Ehrhardt et al., 2004). Similar changes would be expected in canine babesiosis, as falciparum malaria has clinical similarities to canine babesiosis (Maegraith et al., 1957; Jacobson and Clark, 1994).

The primary objective of this study was to determine plasma NT-proBNP concentrations in dogs with mild uncomplicated, severe uncomplicated and complicated 
babesiosis. The secondary objective was to determine if NT-proBNP levels were correlated with myocardial damage, determined by cTnl.

\section{Materials and Methods}

\subsection{Model}

The Ethics and Research Committees of the Faculty of Veterinary Science, University of Pretoria, approved this study and written consent by the dogs' owners was obtained. Forty-five dogs of any breed or sex and weighing more than $3 \mathrm{~kg}$ that were diagnosed at the Onderstepoort Veterinary Academic Hospital (OVAH) with babesiosis were used in this study. Babesiosis was diagnosed on a thin capillary blood smear, stained with Cams Quick stain (CA Milsch, Krugersdorp, South Africa). Exclusion criteria were any clinical signs that could not be ascribed to babesiosis and dogs with known or suspected concurrent cardiac disease of other causes. Concurrent hemoparasites were determined by blood smear examination. On a few cases selected cases PCR was performed (data not shown) and was negative for other hemoparasites. In 3 cases the $B$. rossi parasitemia was also confirmed by sequencing.

Three groups of babesiosis cases were identified: mild uncomplicated (Group 1), severe uncomplicated (Group 2), and complicated (Group 3), with 15 dogs in each of the groups. Mild cases had mild to moderate anemia (hematocrit $\geq 0.20 \mathrm{I} / \mathrm{l}$ ) with no clinical or biochemical signs of complicated disease. Severe uncomplicated cases had severe anemia (hematocrit $<0.20 \mathrm{I} / \mathrm{l}$ ) with no clinical or biochemical signs of complicated disease. Complicated cases showed one or more of the following complications: cerebral signs (ataxia, seizures, unconscious vocalization), immune-mediated hemolytic anemia (IMHA), oliguria or azotemia that did not respond to fluid therapy indicating acute renal failure (ARF), 
bleeding tendency (epistaxis, petechia), severe icterus, or respiratory distress that was not associated with anemia, indicating possible ARDS. These complications were diagnosed clinically and aided by further diagnostic procedures in most of the hospitalized cases. IMHA was diagnosed on a positive in-saline agglutination test. Fifteen dogs that were presented to the OVAH for routine sterilization were used as controls (Group 4).

\subsection{Laboratory measurements}

Excluding blood pressure determination and the echocardiography fractional shortening (FS), all data was collected at presentation and prior to any therapeutic intervention. Blood pressure determination and the FS were performed within 8 hours of admission. Two EDTA tubes, 1 serum tube, and $1 \mathrm{ml}$ of heparinised blood were collected from the jugular vein from all dogs at presentation. One of the EDTA tube was centrifuged within 5 minutes) and the plasma stored at $-80^{\circ} \mathrm{C}$, until the NT-ProBNP assays were done. Sample collection and storage was done over 2 years. The other EDTA and the heparinised sample were used for immediate hematology and blood lactate assay, respectively.

From each animal the following parameters were determined: complete blood count (CBC), blood smear analysis, urine analysis (specific gravity, dipstick and urine sediment), serum urea, creatinine, cystatin-C, and cTnl, blood lactate, plasma NT-proBNP, FS, and blood pressure. Serum urea, creatinine and cystatin-C levels were measured to evaluate renal function as impaired renal function may lead to delayed excretion of circulating cardiac biomarkers (Boswood et al., 2008). The urea: creatinine (UC) ratio was calculated as myocardial injury has been shown to increase the ratio (Feinfeld et al., 2002). As lactic acidosis and poor perfusion have been shown to impair cardiac function and integrity, blood lactate and blood pressure were also evaluated in this study.

CBC was conducted on a Cell Dynn 3500 analyzer (Abbott Laboratories, Abbott Park, 
Illinois, USA). Serum urea and creatinine were determined on a Technicon RA 1000 system (Abbott Laboratories, Abbott Park, Illinois, USA). Blood lactate was measured using the Accusport blood lactate analyzer (Roche Products, Africa Region, Box 129, Isando, South Africa). Serum cystatin-C concentration was measured using the particle-enhanced turbidimetric immunoassay, according to manufacturer's instructions (Diagnostech, Honeydew Dako Cytomation, Cystatin C PET kit, Denmark). Cardiac troponin I concentrations were determined with a solid-phase, enzyme-labeled chemiluminescent immunometric assay (Immulite 2000 Troponin I, Siemens Medical Solutions Diagnostics, 5210 Pacific Concourse Drive, Los Angeles, California, USA) using cTnl-specific purified, polyclonal murine and caprine antibodies with independent immunologic epitopes for cTnl. The detection limit was $0.2 \mathrm{ng} / \mathrm{ml}$. The upper limit of normality for the cTnl used in this study was $1 \mathrm{ng} / \mathrm{ml}$, which was based on the cut-off value used in humans and canine studies (Lobetti et al., 2002; Schober et al., 1999). Plasma NT-proBNP was determined using a commercially available canine-specific NT-proBNP enzyme immunoassay (Cardiopet $^{\circledR}$ ProBNP, Idexx Laboratories, Johannesburg, South Africa) according to manufactures instructions, with values less than $210 \mathrm{pmol} / \mathrm{l}$ considered negative for heart disease, between 210 and 300 a probability of heart disease, and over 300 indicative of heart disease). For both NT-proBNP and cTnl samples, controls, and standards were performed in duplicate

Echocardiography was performed with an Omnia Sonoline ultrasound machine (Siemens, Germany) using a $5 \mathrm{MHz}$ phased array transducer. Fractional shortening was calculated from M-mode measurements of the left ventricle and was an average of 3 measurements.

Blood pressure was determined non-invasively by an oscillometric technique, using a Dinamap blood pressure monitor (Critikon, Johnson \& Johnson, Medical Road, Halfway House, South Africa) and neonatal cuffs (Disposa-Cuf, Critikon), and was an average of 5 measurements. Values greater than $160 \mathrm{mmHg}$ and lower than $80 \mathrm{mmHg}$ were considered to 
be hypertension and hypotension, respectively.

\subsection{Data analysis}

All data were tabulated in a spreadsheet program (Excel ${ }^{\circledR}$ ) and statistical analysis was performed with the aid of a statistical software package (NCSS ${ }^{\circledR}, 329$ North 1000 East, Kaysville, Utah, USA). Descriptive statistics were used to describe the data. One-way analysis of variance with Bonferroni and Tukey-Kramer comparisons was used to test for statistical differences between groups. The Pearson Product correlation was used to determine correlation between variables. The level of significance was set at $p<0.05$.

\section{Results}

The median age in Group 1 was 24 months (range 7-129); Group 2, 24 months (range 6-92); Group 3, 12 months (range 3.5-60) and Group 4, 13 months (range 4-81). Groups 3 and 4 were statistically younger that the other 2 groups. The median weight in Group 1 was 19.5 kg (range 5.6-52); Group 2, 7.6 kg (range 5.1 - 26), Group 3, 18.7 kg (range 6.8 - 43.4) and Group 4, $8 \mathrm{~kg}$ (range 5.4-40); with groups 2 and 4 statistically lighter than the other 2 groups The complications reported in group 3 included severe icterus (6), IMHA (5), cerebral signs (1), ARDS (2), and hemoconcentration [elevated hematocrit and collapsed state, also called "red babesiosis" (2)] with a number of dogs having more than one complication.

The NT-proBNP results are illustrated in Figure 1. The median value in Group 1 was 246 pmol/l (range 8.5-683); Group 2, 650 pmol/l (range 260-2028); Group 3, 638 pmol/l (range 310-2214) and Group 4, $106 \mathrm{pmol} / \mathrm{l}$ (range 0-248). All 3 babesiosis groups had a statistically significant elevated NT-proBNP level compared to the control group and Groups 
2 and 3 showed a statistically significant difference from Group 1. There was no statistical difference between groups 2 and 3. In Group 1, 6/15 (40\%) dogs had values below 210 pmol/l, $2 / 15(13.3 \%)$ a value between 210 and 300 , and $7 / 15(46.7 \%)$ had values above 300 . In Group 2, two of the dogs (13\%) had a value between 210 and 300 whereas the remaining $13 / 15$ dogs $(87 \%)$ were above 300 . All dogs in group 3 had values greater than $300 \mathrm{pmol} / \mathrm{l}$.

The cTnl results are illustrated in Figure 2. In Group 1, 6/15 (40\%) dogs had cTnl concentrations below the detection limit $(0.2 \mathrm{ng} / \mathrm{ml}), 4 / 15(26.7 \%)$ showed a concentration below the upper limit of the reference range $(1 \mathrm{ng} / \mathrm{ml})$, and $5 / 15(33.3 \%)$ had elevated concentrations. The median for group 1 was 0.39 and the range 0.2-9.19. In Group 2, 7/15 $(46.7 \%)$ had concentrations below the detection limit, $3 / 15(20 \%)$ showed a concentration below the upper limit of the reference range, and $5 / 15$ (33.3\%) had elevated concentrations. The median for the group was $0.4 \mathrm{ng} / \mathrm{ml}$ and the range $0.2-21.9$. In Group 3, $1 / 15(6.7 \%)$ had concentrations below the detection limit, $6 / 15(40 \%)$ had concentrations below the upper limit of the reference range, and $8 / 15(53.3 \%)$ had elevated concentrations. The median for the group was $1.45 \mathrm{ng} / \mathrm{ml}$ and the range $0.2-14.4$. All dogs in the control group had concentrations below the detection limit. There was a statistically significant difference in cTnl concentration between the control group and group 3. There was no statistically significant difference between the babesiosis groups.

The median FS in Group 1 was 33.7\% (range 23.2-45.5); Group 2, 43.9\% (range 23.3-51.7); Group 3, 38.9\% (range 19.4-59.2) and Group 4, 37.8\% (range 18.4-49.6). Although there was no statistical significance between the groups, it would appear that both groups 2 and 3 tended towards a higher FS.

Median systolic blood pressure in Group 1 was 137 mmHg (range 109-179); Group 2, 134 (range 105-161); Group 3, 143 (range 87-180) and Group 4, 145 (range 106-183); with no statistical significance between the groups. There were, however, 5 individual hypertensive animals - 2 in group 1, and 1 in each of groups 2, 3, and 4; but no hypotensive 
animals. Median diastolic blood pressure in Group 1 was 89 (range 42-118); Group 2, 73 (range 34-94); Group 3, 76 (range 27-127) and Group 4, 84 (range 55-162); with no statistical significance between the groups. Median mean blood pressure in Group 1 was 103 (range 47-127); Group 2, 91 (range 59-119); Group 3, 99 (range 48-152) and Group 4, 106 (range 83-167); with no statistical significance between the groups.

Median blood lactate in Group 1 was 2.6 mmol/l (range 1.3-4.2); Group 2, 2.3 (range 0.8-22); Group 3, 2.95 (range 1.9-22) and Group 4, 1.9 (range 1.3-2.8); with no statistical significance between the groups.

The median hematocrit in Group 1 was 0.34 I/I (range 0.21-0.47); Group 2, $0.11 \mathrm{I} / \mathrm{I}$ (range 0.7-0.18); Group 3, $0.18 \mathrm{I} / \mathrm{I}$ (range 0.7-0.47) and Group 4, $0.46 \mathrm{I} / \mathrm{I}$ (range 0.36-0.58). All 3 babesiosis groups had a statistically significant lower hematocrit than the control group and Groups 2 and 3 showed a statistically significant difference from Group 1.

All 4 groups showed an appropriate urine specific gravity with all groups having a median greater than 1.035. The median serum creatinine in Group 1 was $93 \mu \mathrm{mol} / /$ (range 44-139); Group 2, $64 \mu \mathrm{mol} / \mathrm{l}$ (range 31-109); Group 3, $123 \mu \mathrm{mol} / \mathrm{l}$ (range 37-260) and Group $4,82 \mu \mathrm{mol} / \mathrm{l}$ (range 51-124), with group 3 showing a statistical significant difference from the other groups. No dogs in group 1 and 2 had elevated serum creatinine and only 2 dogs in group 3 had a serum creatinine higher than the reference range. The median serum urea in Group 1 was $7.1 \mathrm{mmol} / \mathrm{l}$ (range 2.6-17.2); Group 2, 10 mmol/l (range 3.6-31.3); Group 3, $25.6 \mathrm{mmol} / \mathrm{l}$ (range 8.9-48.1) and Group 4, $4.9 \mathrm{mmol} / \mathrm{l}$ (range 2.7-9.1), with group 3 showing a statistical significant difference from the other groups. The median cystatin- $C$ in Group 1 was 1.02 mg/l (range 0.83-1.31); Group 2, 0.92 (range 0.77-2.32); Group 3, 1.61 (range 0.86.62) and Group 41.13 (range 0.83-2.56); with group 3 showing a statistical significant difference from the other groups.

The median UC ratio in Group 1 was 9.6 (range 4.67-40.4); Group 2, 24.51 (range 5.52-53.6); Group 3, 21.41 (range 9.72-45.02) and Group 4, 6.63 (range 4.32-20.51). 
Groups 2 and 3 showed a statistically significant difference from Group 1 and Group 4 (Figure 3).

Five dogs in the complicated group were diagnosed with IMHA. There was, however, neither a significant difference nor a trend between the positive and the negative dogs with regards to cTnl, T-proBNP, and hematocrit. There was also no trend with dogs showing specific complications of babesiosis and cTnI, NT-proBNP, and hematocrit. In all babesiosis groups there was no correlation between the NT-proBNP and hematocrit, cTnl, FS, urea, creatinine, and lactate. Another correlation done was between the pulse and haematocrit, which showed no correlation.

All dogs recovered after treatment.

\section{Discussion}

The results of this study showed that dogs with babesiosis had elevated NT-proBNP and that the elevation was proportional to the severity of the disease. This is the first study to report such findings. NT-proBNP was selected to see if it could determine the presence of functional cardiac dysfunction before there was biochemical evidence on myocardial damage (cTnl), as histopathology was not undertaken in this study. The study showed that elevated NT-proBNP appeared to occur earlier and was independent of myocardial damage, detected by cTnl. From this study it would appear that in canine babesiosis there are functional cardiac changes before myocardial damage is evident. In babesiosis the diagnosis of heart disease is compounded by the presence of the anemic hypoxic state. The early and accurate diagnosis of heart dysfunction in dogs with babesiosis would ensure early and appropriate management. A similar situation occurs in humans with heart failure in that early diagnosis enables patients to be managed rapidly and for this reason, there has been 
significant interest in the development of tests for biomarkers of heart disease and failure in humans patients (Boswood et al., 2008).

In one study (Charpentier et al., 2004), humans with sepsis and/or septic shock, systolic myocardial dysfunction with transient hypotension was a common finding. The study also showed that NT-proBNP levels were useful to detect myocardial dysfunction early in the clinical course of sepsis and persistence of high NT-proBNP levels significantly increased the risk of death. In our study serial NT-proBNP was not done, thus no conclusion could be established if a similar situation exists in dogs with babesiosis, but the once off elevated NTproBNP support makes such a scenario highly suspected. Furthermore none of the dogs showed hypotension, however, blood pressure was only determined within 8 hours after admission and thus transient hypotension may have been missed.

One study concluded that although the prognostic interest of NT-proBNP was superior to other tested variables its use as a prognostic indicator might have been improved by serial NT-proBNP monitoring (Serres et al., 2009). Similarly this can be applied to dogs with babesiosis. Numerous human studies have demonstrated the ability of natriuretic peptides to predict outcomes in patients with various cardiac diseases (Bettencourt, 2004). Similarly, natriuretic peptides have been widely demonstrated to be useful as markers of the presence and severity of canine cardiac disease (Boswood et al., 2003; Boswood et al., 2008; Fine et al., 2008; Haggstrom et al., 2000; Oyama et al., 2008; Prosek et al., 2007). In a study of 330 dogs presented with cough or dyspnea, showed that, although NT-proBNP concentrations were remarkably lower than in humans, NT-proBNP was able to discriminate between heart failure and non-heart failure as a cause for the clinical signs (even in dogs with a previous medical history of heart failure) and also correlated with severity of heart failure (De Francesco et al., 2007). In a study in dogs with mitral valve disease, NT-proBNP was shown to be an aid in the evaluation of the symptomatic phase and provided information on both disease severity and prognosis (Serres et al., 2009). 
Cardiac troponin has been shown to be the most sensitive and specific factor for the prediction of myocardial injury and in humans it can be detected from about 8 hours after the cardiac injury and 10 days thereafter (Collinson et al., 2000; Lee et al., 2008), an ideal time window for clinical canine babesiosis that are usually represent within this time frame after the infection started. In our study approximately $33 \%$ of dogs with mild and severe babesiosis had elevated cTnl whereas the number increased to $53 \%$ in the complicated group. This is in agreement with previous studies where the magnitude of elevation was proportional to the severity of the disease (Dvir et al., 2004; Lobetti et al., 2002). There was no correlation between cTnl and hematocrit and therefore unlikely that anemia caused myocardial hypoxia. As babesiosis is a hemolytic disease a severe drop in hemoglobin can occur. Several animal studies have indicated that at a hematocrit of $0.1-0.15-\mathrm{I} / \mathrm{l}$ tissue oxygenation is maintained (Weisekopf, 1995). The critical value of hemoglobin at which oxygen delivery is equal to oxygen utilization has been shown to occur at a hematocrit of approximately 10\% (van der Linden et al., 1998). Below these levels of critical oxygen delivery, signs of circulatory collapse and shock develop. In pigs signs of shock include reduction in cardiac output, increased central venous pressure, bradycardia, decreased mixed venous saturation, arterial hyperlactatemia, and hypotension (Schou et al., 1998). In this study a reduction in cardiac output (as determined by FS), bradycardia, hyperlactatemia, or hypotension was not evident, making shock an unlikely cause. Furthermore as there was no correlation between lactate and hematocrit it would suggest that any increase in lactate would more likely reflect changes in perfusion and/or cardiac output rather than lower oxygen carrying capacity. As expected, dogs with babesiosis had a low hematocrit, however, this did not appear to influence the cardiac biomarkers, as there was no correlation with NTproBNP and cTnl, and FS. As a dog becomes more anemic, it would be expected that heart rate and thus peripheral pulse would increase as a compensatory mechanism. There was, however, no correlation between pulse and hematocrit in this study. 
Although renal dysfunction can elevate NT-proBNP, in this study there was no correlation with the urea and creatinine and serum NT-proBNP values. Only 2/15 dogs in the complicated group showed azotemia, one with evidence of acute kidney damage, which did not have a severe elevation in NT-proBNP. An increased serum creatinine concentration has been associated with an increased NT-proBNP concentration (Boswood et al., 2008), which was not evident in this study. One study showed that dogs with renal disease had significantly higher mean serum concentration of NT-proBNP than control dogs (Schmidt et al., 2009). Renal function should be considered when interpreting NT-proBNP results as concentrations may be falsely elevated in dogs with renal dysfunction and normal cardiac function.

In this study, dogs with complicated babesiosis showed a disproportionate increased urea with respect to creatinine. This group, as well as the dogs with severe babesiosis, also showed an elevated UC ratio, indicative of pre-renal azotemia. Azotemia, in the form of elevated serum urea concentrations, is common in canine babesiosis (Reyers, 1992; Reyers et al, 1998). In a retrospective canine babesiosis study, the median serum urea concentration was approximately double the normal laboratory range, whilst the median serum creatinine concentration fell within the normal range (De Scally et al., 2004). In humans, cardiac disease has been reported to be a major contributor of an increased serum UC ratio (Feinfield et al., 2002). As significant myocardial disease has been reported in canine babesiosis (Dvir et al., 2004; Lobetti et al., 2002), myocardial failure may account for the increased serum UC ratio in canine babesiosis.

Babesiosis results in various degrees of anemia (Lobetti, 1998), which will result in volume retraction and high-output states (increased preload, reduced afterload) and can result in high output heart failure. The volume overload can result in increased stretch and thus an increase in NT-proBNP. This study suggests that dogs with severe babesiosis suffer from cardiac volume overload, as this is the major cause of elevated NT- 
proBNP. Such elevated levels originate most probably from myocardial failure, however some of the increase can also secondary to pulmonary complications (De Lemos et al., 2001) that have been described in canine babesiosis (Lobetti, 1998). In such cases the "cut off" for diagnosis of heart involvement might need to be increased (De Lemos et al., 2001). Although pulmonary complications occur in babesiosis (Lobetti, 1998), in this study only two dogs showed pulmonary oedema (ARDS), without their NT-proBNP being severely elevated. In the other dogs the presenting sign of polypnea resolved with parasite therapy and blood transfusion and none of these dogs went on to develop pulmonary complications. It is, obviously, warranted to investigate further the interrelation between the pulmonary and the cardiovascular complications in babesiosis.

The only cardiac variable assessed in this study was FS and it was determined a number of hours after the diagnosis had been made and often after therapeutic interventions had been done, thus potentially changing conventional echocardiographic variables. Fractional shortening is the most common and easily aquired measuremnt of ventricular function and may be affected by preload, afterload and contractility (Boone 2011). In an experimentally induced acute normovolemic anemia study in conscious dogs by our babesiosis study group, which mimicked severe uncomplicated babesiosis, the increased FS was similar to the severe uncomplcated group in the current study (FS of $47.3 \%$ with a $\mathrm{Ht}$ of $0.15 \mathrm{l} / \mathrm{l}$ and $44 \%$ with a $\mathrm{Ht}$ of $0.11 \mathrm{l} / \mathrm{l})$ (Spotswood et al., 2006). This increased fractional shortening is indicative of increased cardiac output secondary to the hyperdynamic state that exists in severely anemic patients which improves as the anemic state improves (Spotswood et al., 2006). Increased cardiac output is regarded as the most important compensatory mechanism in maintaining adequate tissue oxygenation, particularly in the face of moderate to severe chronic anemia where the other mechanisms are already saturated (Varat et al., 1972). An extensive echocardiographic examination in our critically ill groups was not deemed feasible for this study and hence only FS was determined as a bedside test to get 
some idea of cardiac function. Based on the experimental study mentioned previously it does not appear that the expected cardiac function changes seen in anemia were at all altered by the presence of babesiosis and that the elevated NT-proBNP most likely resulted from the increased preload with associated myocardial stretch.

In a previous study (Lobetti et al, 2002), an association between cTnl concentration and histological changes, clinical severity, and survival was evidenced in dogs with babesiosis. The present study seems to indicate that NT-proBNP could be a more appropriate marker for early detection of cardiac dysfunction, showing an increase with the severity of the disease. The cause-effect relationship, however, remains to be established, as this study did not demonstrate that the elevated NT-proBNP was associated with occurrence and development of cardiac lesions and/or cardiac dysfunction. Thus it can only be hypothesized that the elevations in NT-proBNP with increasing severity of babesiosis indicates presence and development of cardiac dysfunction. The lack of relationships between cTnl and NT-proBNP would indicate that the cardiac dysfunction (inducing the increase in NT-proBNP), if any, is independent of the development of cardiac lesions (demonstrated in this study by increased cTnl). 


\section{References}

Asano, K., Masuda, K., Okumura, M., Kadosawa, T., Fujinaga, T. 1999. Plasma atrial and brain natriuretic peptide levels in dogs with congestive heart failure. J. Vet. Med. Scien. 61,523-529.

Bettencourt, P. 2004. NT-proBNP and BNP: biomarkers for heart failure management. Eur. J. Heart Fail. 6,359-363.

Boone, J.A. 2011. Veterinary Echocardiography. $2^{\text {nd }}$ ed. Wiley Blackwell, Ames lowa. $p 201$.

Boswood, A., Attree, S., Page, K. 2003. Clinical validation of a proANP 31-67 fragment ELISA in the diagnosis of heart failure in the dog. J. Smal. Anim. Prac. 44,104-108.

Boswood, A., Dukes-McEwen, J., Loureiro, J., James, R.A., Martin, M., Stafford-Johnson, M., Smith, P., Little, C., Attree, S. 2008. The diagnostic accuracy of different natriuretic peptides in the investigation of canine cardiac disease. J. Smal. Anim. Prac. $49,26-32$.

Charpentier, J., Luyt, C.E., Fulla, Y. 2004. Brain natriuretic peptide: A marker of myocardial dysfunction and prognosis during severe sepsis. Crit. Care. Med. 32,660 -665.

Chetboul, V., Tessier-Vetzel, D., Escriou, C., Tissier, R., Carlos, C., Boussouf, M., Pouchelon, J.L., Blot, S., Derumeaux, G. 2004. Diagnostic potential of natriuretic peptides in the occult phase of golden retriever muscular dystrophy cardiomyopathy. J Vet Intern Med 18,845-850.

Collinson, P.O., Premachandram, S., Hashemi, K. 2000. Prospective audit of incidence of prognostically important myocardial damage in patients discharged from emergency department. Brit. Med. J. 320,1702-1705.

DeFrancesco, T.C., Rush, J.E., Rozanski, E.A., Hansen, B.D., Keene, B.W., Moore, D.T., Atkins, C.E. 2007. Prospective clinical evaluation of an ELISA B-type natriuretic peptide assay in the diagnosis of congestive heart failure in dogs presenting with 
cough or dyspnea. J Vet Intern Med 21,243-250.

De Lemos, J.A., Morrow, D.A., Bentley, J.H., Omland, T., Sabatine, M.S., McCabe, C.H., Hall, C., Cannon, C.P., Braunwald, E. 2001. The prognostic value of B-type natriuretic peptide in patients with acute coronary syndromes. New. Eng. J. Med. $345,1014-1021$.

De Scally, M.P., Lobetti, R.G., Reyers, F., Humphris, D. 2004. Are urea and creatinine values reliable indicators of azotaemia in canine babesiosis? J. South. Afric. Vet. Assoc. $75,121-124$.

Dvir, E., Lobetti, R.G., Jacobson, L.S., Pearson, J., Becker, P.J. 2004. Electrocardiographic changes and cardiac pathology in canine babesiosis. J. Vet. Card. 6,15-23.

Ehrhardt, S., Wichmann, D., Hemmer, C.J., Burchard, G.D., Brattig, N.W. 2004. Circulating concentrations of cardiac proteins in complicated and uncomplicated Plasmodium falciparum malaria. Trop. Med. Internat. Healt. 9,1099-1103.

Ehrhardt, S., Mockenhaupt, F., Anemana, S.D., Otchwemah, R.N., Wichmann, D., Cramer, J.P., Bienzle, U., Burchard, G.D., Brattig, N.W. 2005. High levels of circulating cardiac proteins indicate cardiac impairment in African children with severe Plasmodium falciparum malaria. Microb. Infect. 7,1204-1210.

Feinfeld, D.A., Bargouthi, H., Niaz, Q., Carvounis, C.P. 2002. Massive and disproportionate elevation of blood urea nitrogen in acute azotaemia. Intern. J. Urol. Neph. 34,143145.

Fine, D.M., Declue, A.E. \& Reinero, C.R. 2008. Evaluation of circulating amino terminal-proB-type natriuretic peptide concentration in dogs with respiratory distress attributable to congestive heart failure or primary pulmonary disease. J. Am. Vet. Med. Assoc. 232,1674-1679.

Fonfara, S., Loureiro, J., Swift, S., James, R., Cripps, P., Dukes-McEwan, J. 2010. Cardiac troponin I as a marker for severity and prognosis of cardiac disease in dogs. Vet. J. 
$184,334-339$

Haggstrom, J., Hansson, K., Kvart, C., Pedersen, H.D., Vuolteenaho, O., Olsson, K. 2000. Relationship between different natriuretic peptides and severity of naturally acquired mitral regurgitation in dogs with chronic myxomatous valve disease. J. Vet. Card. 2,716.

Jacobson, L.J., Clark, I. 1994. The pathophysiology of canine babesiosis: new approaches to an old puzzle. J. South Afric. Vet. Assoc. 65,134-145.

Jacobson, L.S., Lobetti, R.G., Vaughan-Scott, T. 2000. Blood pressure changes in dogs with babesiosis. J. South. Afric. Vet. Assoc. 71,14-20.

Lee, H.S., Son, C.B., Shin, S.H., Kim, Y.S. 2008. Clinical correlation between brain natriutetic peptide and anthracyclin-induced cardiac toxicity. Can. Res. Treat. 40,121126.

Lobetti, R.G. 1998. Canine babesiosis. Comp. Cont. Educ. Pract. Vet. 20,418-430.

Lobetti, R.G., Dvir, E., Pearson, J. 2002. Cardiac troponins in canine babesiosis. J Vet. Intern. Med. 16,63-68.

Maegraith, B., Gilles, H.M., Devakul, K. 1957. Pathological process in Babesia canis infections. Zeitschrift für Tropenmedizin und Parasitologie $8,485-514$.

Missov, E., Calzolari, C., Pau, B. 1997. Circulating cardiac troponin I in severe congestive heart failure. Circulation. 96,2953-2958.

O'Brien, P.J., Landt, Y., Ladenson, J.H. 1997. Differential reactivity of cardiac and skeletal muscle from various species in a cardiac troponin I immunoassay. Clin. Chem. 43,2333-2338.

Omland, T., Aakvaag, A., Bonarjee, V.V.S., Caidahl, K., Lie, R.T., Nilsen, D.W., Sundsfjord, J.A., Dickstein, K. 1996. Plasma brain natriuretic peptide as an indicator of left ventricular systolic function and long-term survival after acute myocardial dysfunction. Circulation. 93,1963-1969. 
Oyama, M.A., Sisson, D.D., Solter, P.F. 2007. Prospective screening for occult cardiomyopathy in dogs by measurement of plasma atrial natriuretic peptide, B-type natriuretic peptide, and cardiac troponin-I concentrations. Am. J. Vet. Res. 68,42-47. Oyama, M.A., Fox, P.R., Rush, J.E., Rozanski, E.A., Lesser, M. 2008. Clinical utility of serum N-terminal pro-B-type natriuretic peptide concentration for identifying cardiac disease in dogs and assessing disease. J. Am. Vet. Med. Assoc. 232,1496-1503.

Oyama, M.A., Rush, J.E., Rozanski, E.A., Fox, P.R., Reynolds, C.A., Gordon, S.G., Bulmer, B.J., Lefbom, B.K., Brown, B.A., Lehmkuhl, L.B., Prosek, R., Lesser, M.B., Kraus, M.S., Bossbaly, M.J., Rapoport, G.S., Boileau, J.S. 2009. Assessment of serum Nterminal pro-B-type natriuretic peptide concentration for differentiation of congestive heart failure from primary respiratory tract disease as the cause of respiratory signs in dogs J. Am. Vet. Med. Assoc.235,1319-1325.

Prosek, R., Sisson, D.D., Oyama, M.A., Solter, P.F. 2007. Distinguishing cardiac and noncardiac dyspnea in 48 dogs using plasma atrial natriuretic factor, B-type natriuretic factor, endothelin, and cardiac troponin-I. J. Vet. Intern. Med. 21,238-242.

Reyers, F. 1992. Is azotaemia in canine babesiosis an indication of renal disease? Proceedings of the 9th Faculty Day, University of Pretoria, Faculty of Veterinary Science. 17.

Reyers, F., Leisewitz, A.L., Lobetti, R.G., Milner, R.J., Jacobson, L.S., van Zyl, M. 1998. Canine babesiosis in South Africa: more than one disease. Does this serve as a model for falciparum malaria? Ann. Trop. Med. Parasitol. 92,503-511.

Rossi, R. 1999. Performance characteristics of the cardiac troponin-I method on the DimensionÒ RxL clinical chemistry system with heterogeneous immunoassay. Boehring Technical Bulletin, Newark, Delaware.

Schmidt, M.K., Reynolds, C.A., Estrada, A.H., Prosek, R., Maisenbacher, H.W., Sleeper, M.M., Oyama M.A. 2009. Effect of azotemia on serum N-terminal proBNP 
concentration in dogs with normal cardiac function: A pilot study. J. Vet. Card. 11, S81-S86.

Schober, K.E., Kirbach, B., Oechtering, G. 1999. Non-invasive assessment of myocardial cell injury in dogs with suspected cardiac contusion. J. Vet. Card. 1,17 - 25.

Schou H, Kongstad L, Perez de Sa V, Werner, O., Larsson, A. 1998. Uncompensated blood loss is not tolerated during acute normovolemic hemodilution in anesthetized pigs. Anesth. Analg. 87,786-794.

Serres, F., Pouchelon, J.L., Poujol, L., Lefebvre, H.P., Trumel, C., Daste, T., Sampedrano, C.C., Gouni, V., Tissier, R., Hawa, G., Chetboul, V. 2009. Plasma N-terminal pro-Btype natriuretic peptide concentration helps to predict survival in dogs with symptomatic degenerative mitral valve disease regardless of and in combination with the initial clinical status at admission. J. Vet. Card. 11,103-121.

Spotswood, T.C., Kirberger, R.M., Koma, L.M.P.K., Thompson, P.N., Miller, D.M. 2006. Changes in echocardiographic variables of left ventricular size and function in a model of canine normovolemic anemia. Vet. Radiol. Ultrasound. 47, 358-365.

van der Linden, P., Schmartz, D., de Groote, F., Mathieu, N., Willaert, P., Rausin, I., Vincent, J.L. 1998. Critical haemoglobin concentration in anaesthetized dogs: comparison of two plasma substitutes. Brit. J. Anaesth. 81,556-562.

Varat, M.A., Adolph, R.J., Fowler, N.O. 1972. Cardiovascular effects of anemia. Am. Heart. J. 83,415-426.

Weisekopf, R.B. 1995. Mathematical analysis of isovolaemic hemodilution indicates that it can decrease the need for allogeneic blood transfusion. Trans. 35,37-41. 


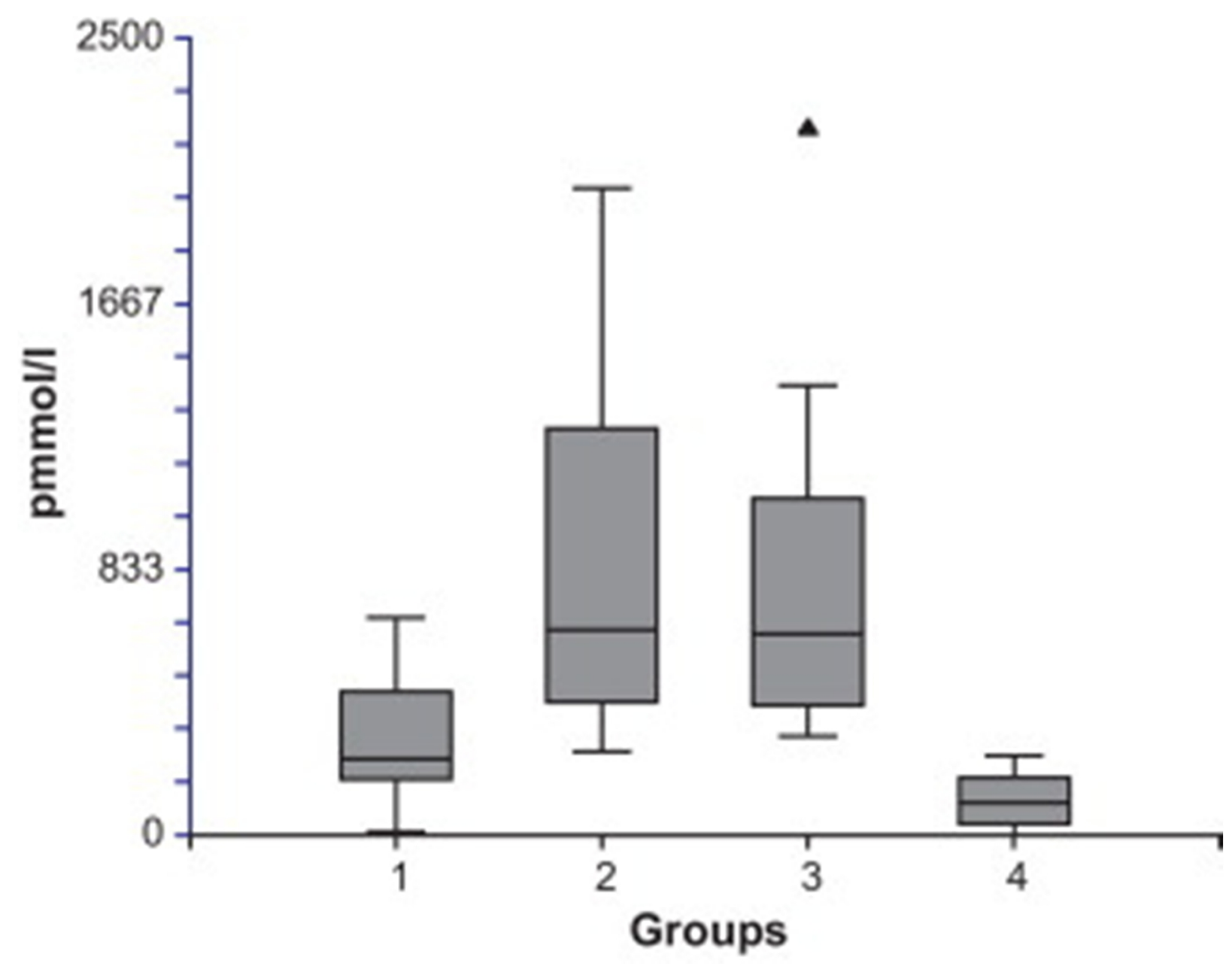

Fig. 1. Plasma NT-proBNP concentrations in 45 dogs with babesiosis and 15 healthy dogs. Data are shown as median (horizontal line within box), 25th and 75th percentiles (horizontal ends of boxes), and 10th and 90th percentiles (T-bars). Black triangle represents an outlier. Group 1 - mild, uncomplicated babesiosis; Group 2 - severe, uncomplicated babesiosis; Group 3 - complicated babesiosis; Group 4 - control. 




Fig. 2. Serum cardiac troponin I concentrations in 45 dogs with babesiosis and 15 healthy dogs. Data are shown as median (horizontal line within box), 25th and 75th percentiles (horizontal ends of boxes), and 10th and 90th percentiles (T-bars). Black triangles represent outliers. Group 1 - mild, uncomplicated babesiosis; Group 2 - severe, uncomplicated babesiosis; Group 3 - complicated babesiosis; Group 4 - control. 


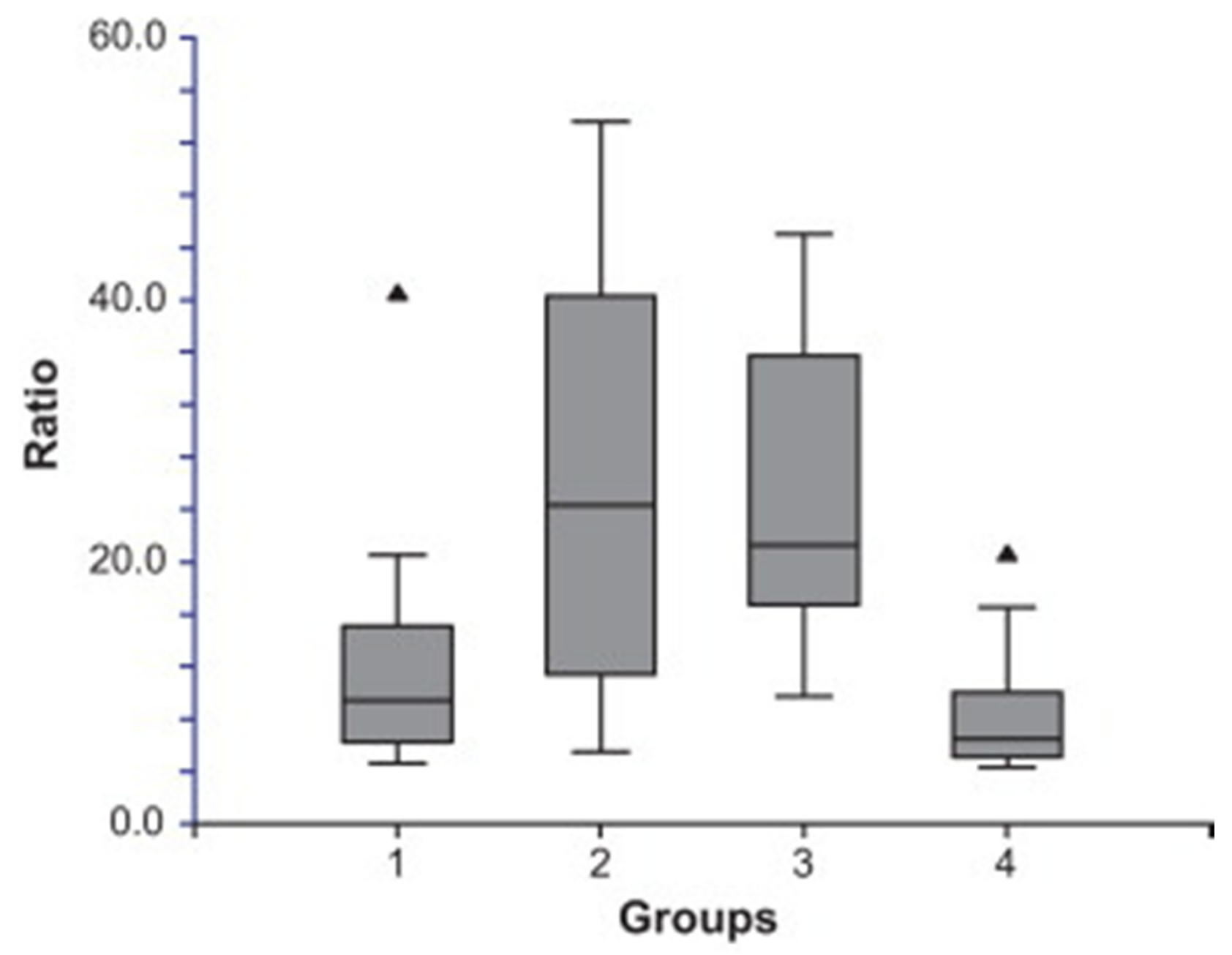

Fig. 3. Urea: creatinine ratioof 45 dogs with babesiosis and 15 healthy dogs. Data are shown as median (horizontal line within box), 25th and 75th percentiles (horizontal ends of boxes), and 10th and 90th percentiles (T-bars). Black triangles represent outliers. Group 1 - mild, uncomplicated babesiosis; Group 2 - severe, uncomplicated babesiosis; Group 3 - complicated babesiosis; Group 4 control. 